\title{
GRB 090426: Discovery of a jet break in a short burst afterglow ${ }^{\star}$
}

\author{
A. Nicuesa Guelbenzu ${ }^{1}$, S. Klose ${ }^{1}$, A. Rossi ${ }^{1}$, D. A. Kann ${ }^{1}$, T. Krühler ${ }^{2,3}$, J. Greiner ${ }^{2}$, A. Rau ${ }^{2}$, F. Olivares E. ${ }^{2}$, \\ P. M. J. Afonso ${ }^{2, \star \star}$, R. Filgas ${ }^{2}$, A. Küpcü Yoldas ${ }^{4}$, S. McBreen ${ }^{5}$, M. Nardini' ${ }^{2}$, P. Schady ${ }^{2}$, S. Schmidl ${ }^{1}$, \\ A. C. Updike ${ }^{6,7,8}$, and A. Yoldaş ${ }^{4}$ \\ 1 Thüringer Landessternwarte Tautenburg, Sternwarte 5, 07778 Tautenburg, Germany \\ e-mail: ana@tls-tautenburg.de \\ 2 Max-Planck-Institut für Extraterrestrische Physik, Giessenbachstraße, 85748 Garching, Germany \\ 3 Universe Cluster, Technische Universität München, Boltzmannstraße 2, 85748, Garching, Germany \\ ${ }^{4}$ Institute of Astronomy, University of Cambridge, Madingley Road CB3 OHA, Cambridge, UK \\ 5 School of Physics, University College Dublin, Dublin 4, Republic of Ireland \\ 6 Clemson University, Department of Physics and Astronomy, Clemson, SC 29634-0978, USA \\ 7 CRESST and the Observational Cosmology Laboratory, NASA/GSFC, Greenbelt, MD 20771, USA \\ 8 Department of Astronomy, University of Maryland, College Park, MD 20742, USA \\ Received 4 February 2011 / Accepted 16 April 2011
}

\section{ABSTRACT}

\begin{abstract}
Context. The link between the duration of GRBs and the nature of their progenitors remains disputed. Short bursts (with durations of less than $\sim 2 \mathrm{~s}$ ) are less frequently observed, technically more difficult to localize, and exhibit significantly fainter afterglows. Aims. It is of critical importance to establish whether the burst duration can reliably distinguish the different GRB population models of collapsars and compact stellar mergers. The Swift GRB 090426 provides an unique opportunity to address this question. Its duration $\left(T_{90}=1.28 \mathrm{~s}\right)$ places GRB 090426 firmly in the short burst population, while the high redshift $(z=2.609)$, host galaxy properties, and prompt emission spectral characteristics are more similar to those of long-duration GRBs.

Methods. On the basis of data obtained with the Tautenburg $2 \mathrm{~m}$ telescope (Germany) and the 7-channel imager GROND (La Silla, Chile), we compiled the most finely sampled light curve available for a short burst optical/NIR afterglow. The light curve was then analysed in a standard fashion. GROND and XRT data were used to determine the broad-band spectral energy distribution of the afterglow across more than three orders of magnitude.

Results. Our data show that a light curve break exists at 0.4 days, which is followed by a steep decay. This light curve decay is achromatic in the optical/NIR bands, and interpreted as a post-jet break phase. The X-ray data do not disagree with this interpretation. Conclusions. The half-opening angle of the suspected jet as well as the luminosity of the optical afterglow provide additional evidence that GRB 090426 is probably linked to the death of a massive star rather than to the merger of two compact objects.
\end{abstract}

Key words. gamma-ray burst: individual: GRB 090426

\section{Introduction}

It is commonly accepted that long GRBs are linked to the corecollapse of massive stars (so-called collapsar events; Woosley \& Bloom 2006) residing in star-forming galaxies, while short bursts are linked to compact stellar mergers in all morphological types of galaxies (Fong et al. 2010; Nakar 2007). Since the launch of the Swift satellite (Gehrels et al. 2004), about 100 long GRBs have been rapidly localized per year (Gehrels et al. 2009). Nearly half of them have a detected optical afterglow and one third have redshift determinations (see J. Greiner's web-page ${ }^{1}$ ). Compared to the long burst sample, short bursts are less frequently observed, and generally followed by on average significantly fainter and less luminous afterglows (Kann et al. 2011, 2010; Nysewander et al. 2009). By the end of 2010, about three dozen short GRBs had been localized by Swift (fewer than

\footnotetext{
* Appendix A is available in electronic form at http: //www . aanda.org

$\star \star$ Present address: American River College, Department of Physics and Astronomy, 4700 College Oak Drive, Sacramento, CA 95841, USA.

${ }^{1}$ http://www .mpe.mpg.de/ jcg/grbgen.html
}

10 events per year). Among them, about 50\% have optical detections and about one third have redshift determinations based on host galaxy spectroscopy (Berger 2010).

GRB 090426 is an outstanding short burst $\left(T_{90} \sim 1.28 \mathrm{~s}\right)$, because it has by far the highest redshift known among the short burst sample $(z=2.609$; Antonelli et al. 2009; Levesque et al. 2010). All other short bursts with secure redshift measurements have $z \lesssim 1.1$ (Berger 2010). The redshift of GRB 090426 is therefore in much closer agreement with the distribution of long GRB redshifts than with that of the short burst sample. Several arguments have been put forward that GRB 090426 is not related to merging compact objects. Antonelli et al. (2009) found that the GRB spectral and energy properties are more comparable to those of collapsar events. Levesque et al. (2010) noted that the blue star-forming host of this burst might also be consistent with a collapsar origin. Similarly, Xin et al. (2010) argued that the deduced lower limit to the circumburst particle number density (about $10 \mathrm{~cm}^{-3}$ ) is much higher than expected for a merging stellar system, being more characteristic of a star-forming region.

We present additional multi-color photometry of the optical/NIR afterglow of GRB 090426 from about 0.3 to 2.5 days 
after the burst, showing that the optical light curve has a welldefined break at late times ${ }^{2}$.

\section{Observations and data reduction}

Observations of the optical/NIR afterglow of GRB 090426 were performed with the $2 \mathrm{~m}$ telescope of the Thüringer Landessternwarte Tautenburg (TLS, Germany) and the 7-band multichannel imager GROND (Greiner et al. 2008) mounted at the $2.2 \mathrm{~m} \mathrm{ESO/MPI} \mathrm{telescope} \mathrm{on} \mathrm{La} \mathrm{Silla} \mathrm{(Chile).}$

Tautenburg started observing in the $I_{\mathrm{c}}$ and $R_{\mathrm{c}}$ band at 19:58 UT on 26 April 2009 and stayed on the field for $90 \mathrm{~min}$. The average airmass was 1.1 and the average seeing 1.2 arcsec. About $3.5 \mathrm{~h}$ after the end of the TLS observations, at 01:08 UT on 27 April 2009 (12.3 h after the GRB trigger), GROND started following the afterglow once the target became visible over La Silla. Observations continued until 04:55 UT at an average seeing of 1.2 arcsec and an average airmass of 2.5, during which GROND was able to detect the afterglow in 10 different $\mathrm{OBs}^{3}$ (Table A.1). Second and third epoch observations were obtained the following two nights.

Data reduction was performed in a standard fashion. TLS and GROND data were analysed through standard PSF photometry using DAOPHOT and ALLSTAR tasks of IRAF (Tody 1993), in a similar way to the procedure described in Krühler et al. (2008) and Yoldaş et al. (2008). Calibrations were performed against the SDSS, using the transformation equations of Lupton ${ }^{4}$ for the TLS data. Magnitudes were corrected for Galactic extinction, assuming $E(B-V)=0.017 \mathrm{mag}$ (Schlegel et al. 1998) and a ratio of total-to-selective extinction of $R_{V}=3.1$.

\section{Results}

\subsection{Spectral energy distribution (SED)}

The host galaxy of GRB 090426 is an extended source (about $2^{\prime \prime}$ ) and the afterglow lies above the brightest part of this galaxy, the N-E knot (Antonelli et al. 2009). For the galaxy as well as this knot, $g^{\prime} r^{\prime} i^{\prime} z^{\prime}$ magnitudes were previously published (Antonelli et al. 2009), but in the NIR bands only upper limits are known (Levesque et al. 2010). Therefore, for the construction of the afterglow SED, the GROND optical bands could be corrected for the contribution of the underlying host galaxy flux, but in the NIR only the maximum possible contribution of host galaxy light could be considered.

Combining the GROND OBs 6 to 10 provides a good signalto-noise ratio for the detection of the optical transient (afterglow plus underlying host galaxy) in $g^{\prime}, r^{\prime}, i^{\prime}, z^{\prime}, J$, and $H$ (Table A.2). Given the upper limits to the $J, H$-band magnitudes of the underlying host, the contribution of host galaxy flux in $J$ and $H$ at this time translates into an increase in brightness of the optical transient by at most $0.1 \mathrm{mag}$ and $0.13 \mathrm{mag}$, respectively. To correct for this contribution, we added 0.1 mag to the $J$ as well as $H$-band data point and also increased the corresponding $1 \sigma$ error by $0.1 \mathrm{mag}$. Performing the fit from $g^{\prime}$ to $H$ then gives $\beta_{\text {opt } / \mathrm{NIR}}=0.76 \pm 0.14$ (Fig. 2 ), which is close to the observed mean for optical/NIR afterglows (about 0.6; cf. Greiner et al. 2011; Kann et al. 2010).

\footnotetext{
2 In the following we use the standard notation for the flux density of the afterglow, $F_{v}(t) \propto t^{-\alpha} v^{-\beta}$.

3 Technical name for a pre-defined observing sequence.

4 http://www.sdss.org/dr7/algorithms/

sdssUBVRITransform.html
}

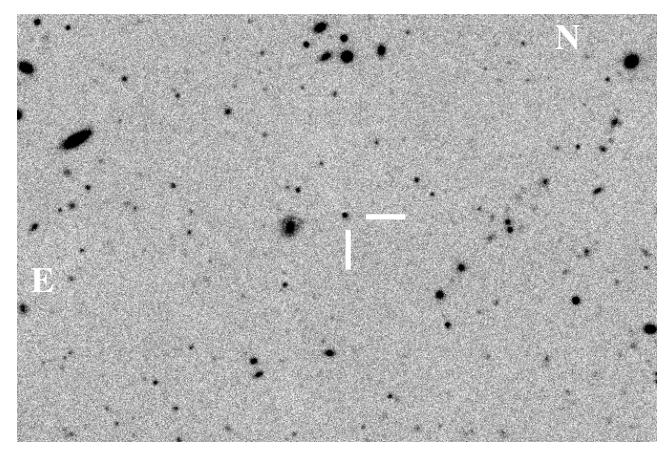

Fig. 1. Finding chart of the afterglow of GRB 090426. The $r^{\prime}$-band GROND image is a combination of OBs 6 to 10 (Table A.1). The field of view is approximately $2^{\prime} \times 3^{\prime}$.

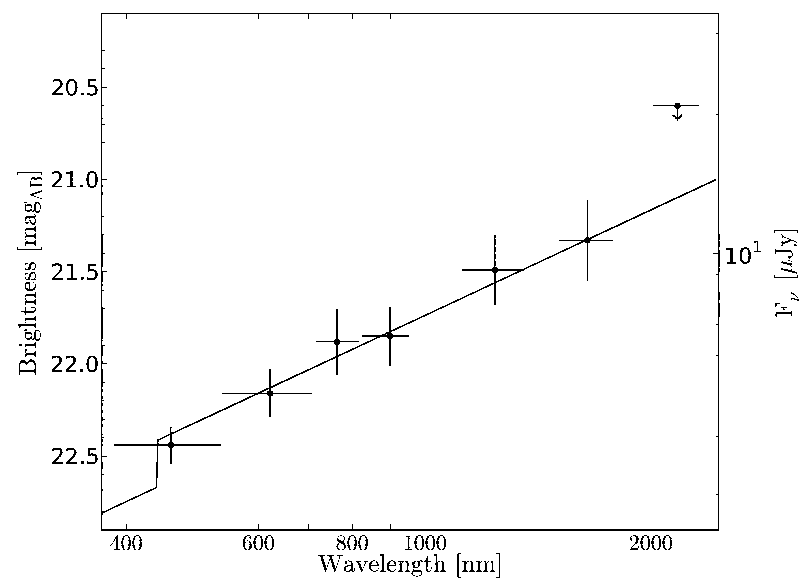

Fig. 2. The SED of the afterglow at a mean time of $t=53 \mathrm{ks}$ (Table A.2). The data is corrected for Galactic extinction and underlying host galaxy contribution. The fit is a single power law $\left(\chi^{2} /\right.$ d.o.f. $\left.=0.17\right)$. There is no evidence of extinction by dust in the GRB host galaxy $\left(A_{V}\right.$ (host) $\left.=0 \mathrm{mag}\right)$. For the fit, the redshift was fixed to $z=2.61$, the Lyman $\alpha$ absorption affects the $g^{\prime}$ band slightly. From left to right, we present results for the $g^{\prime} r^{\prime} i^{\prime} z^{\prime} J H K$ bands.

\subsection{Multi-color light curve}

We combined TLS/GROND data with those of Xin et al. (2010), Levesque et al. (2010), and Antonelli et al. (2009) to obtain an $R_{\mathrm{c}}$-band light curve composed of data sets published in refereed papers. Assuming a power-law SED of the afterglow and a non-evolving spectral index, the $r^{\prime}$-band data were transformed into $R_{\mathrm{c}}$. For completeness, the $V$-band data from Xin et al. (2010) was also used and shifted into the $R_{\mathrm{c}}$ band. The final $R_{\mathrm{c}}$-band data set after the first break at about 0.05 days can be fit using a single broken power law (Beuermann et al. 1999; Fig. 3).

Fitting the data we find a late break in the light curve at around 0.4 days, in addition to a first break at around 0.02 days that had been previously known. This second break in the light curve was not seen in the previous data sets of GRB 090426. The $g^{\prime} r^{\prime} i^{\prime} z^{\prime} J H$ GROND data show that this evolutionary phase is achromatic within the optical/NIR bands (Fig. 3). The most obvious interpretation is that this is a jet break.

\section{Discussion}

\subsection{The light curve parameters}

Jet breaks are widely studied features of long GRB afterglow light curves (cf. Frail et al. 2001). In their long burst sample, Zeh et al. (2006) find a nearly log-normal distribution of jet half-opening angles between 2 and 12 degrees, with the peak 


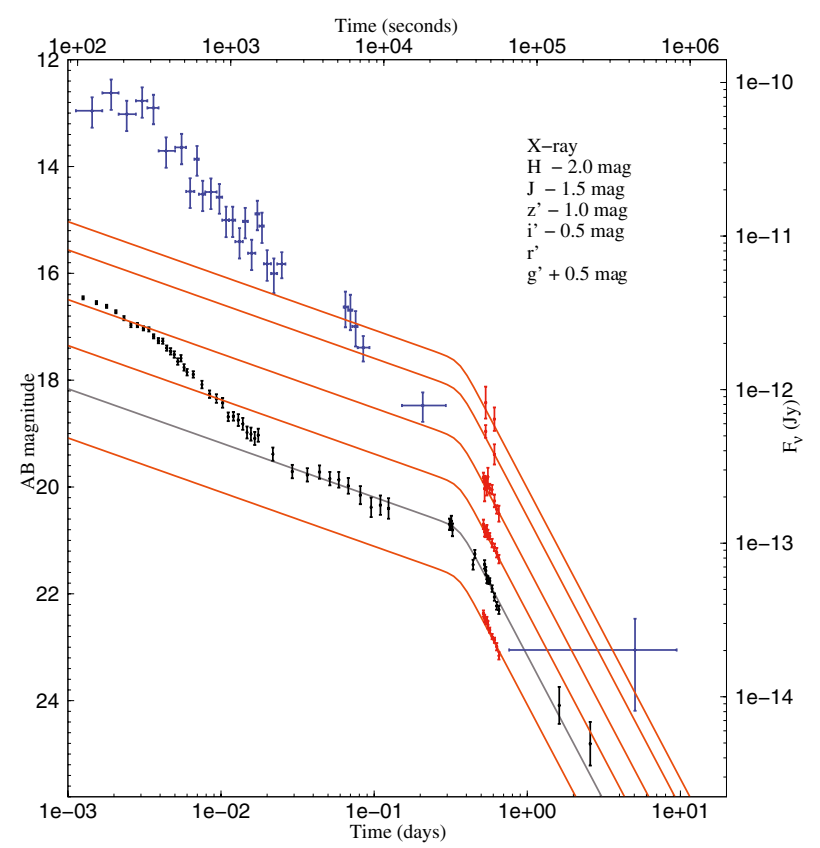

Fig. 3. $R_{\mathrm{c}}$-band light curve of the GRB 090426 afterglow and best fit with a single broken power law after host galaxy subtraction and correction for Galactic extinction. The fit starts at 0.05 days. For comparison, the $g^{\prime} i^{\prime} z^{\prime} J H$ bands (left $y$-axis; Tables A.1-A.3) and the X-ray light curve ( 0.3 to $10 \mathrm{keV}$, Evans et al. 2010; right $y$-axis) are also shown.

around 2 to 4 degrees (see also Racusin et al. 2009). For short GRBs, however, afterglow light curves are typically sparsely sampled. Some cases seem uncollimated (Grupe et al. 2006), while others display breaks and steep late slopes, which are evidence of collimation (Burrows et al. 2006; McBreen et al. 2010; Soderberg et al. 2006). Most of these results, however, rely on the corresponding X-ray light curve. In the case of GRB 090426 , on the other hand, there are basically no X-ray data available around the time of the late break in the optical light curve. The available data seem to indicate a smooth X-ray afterglow decay from $4000 \mathrm{~s}$ on up to the last X-ray detection at about $4 \times 10^{5} \mathrm{~s}$ (Xin et al. 2010). However, a break in the X-ray light curve at 0.4 days, which is simultaneous with the break in the optical bands, is not ruled out.

A satisfying fit of the entire optical and X-ray light curve can be obtained by assuming a two-component jet model (Berger et al. 2003; Peng et al. 2005; Racusin et al. 2008; Filgas et al. 2011; Fig. 4). Within this framework, the observed afterglow light curve is the superposition of the radiation from two jets, a narrow and a wide jet. Even though it was not the aim of this paper to explore the validity of this model for GRB 090426, we used it to fit the data and to shift X-ray and optical data points to the same time after the burst in order to obtain the SED from the optical to the X-ray band.

According to the best fit, the narrow-jet component is described by a single broken power law with $\alpha_{1}=0.48 \pm 0.04$, $\alpha_{2}=1.22 \pm 0.05$, and a break time $t_{b 1}=290 \pm 20 \mathrm{~s}$ (while fixing the smoothness parameter $n_{1}$ to 3 ). The second, wider component, follows a double broken power law with $\alpha_{4}=0.46 \pm 0.15$, $\alpha_{5}=2.43 \pm 0.19$ and break times $t_{b 2}=9400 \pm 3800 \mathrm{~s}$ $(0.11 \pm 0.04$ days $)$ and $t_{b 3}=34500 \pm 1800 \mathrm{~s}(0.39 \pm 0.02$ days; by requiring $\alpha_{3}=-0.5$, Panaitescu $\&$ Kumar 2000, $n_{2}=10$, and $n_{3}=10 ; \chi^{2} /$ d.o.f $\left.=90.39 / 78=1.16\right)$. The optical and the X-ray light curve trace each other, implying that both are belonging to the same spectral regime. We caution, however, that after about

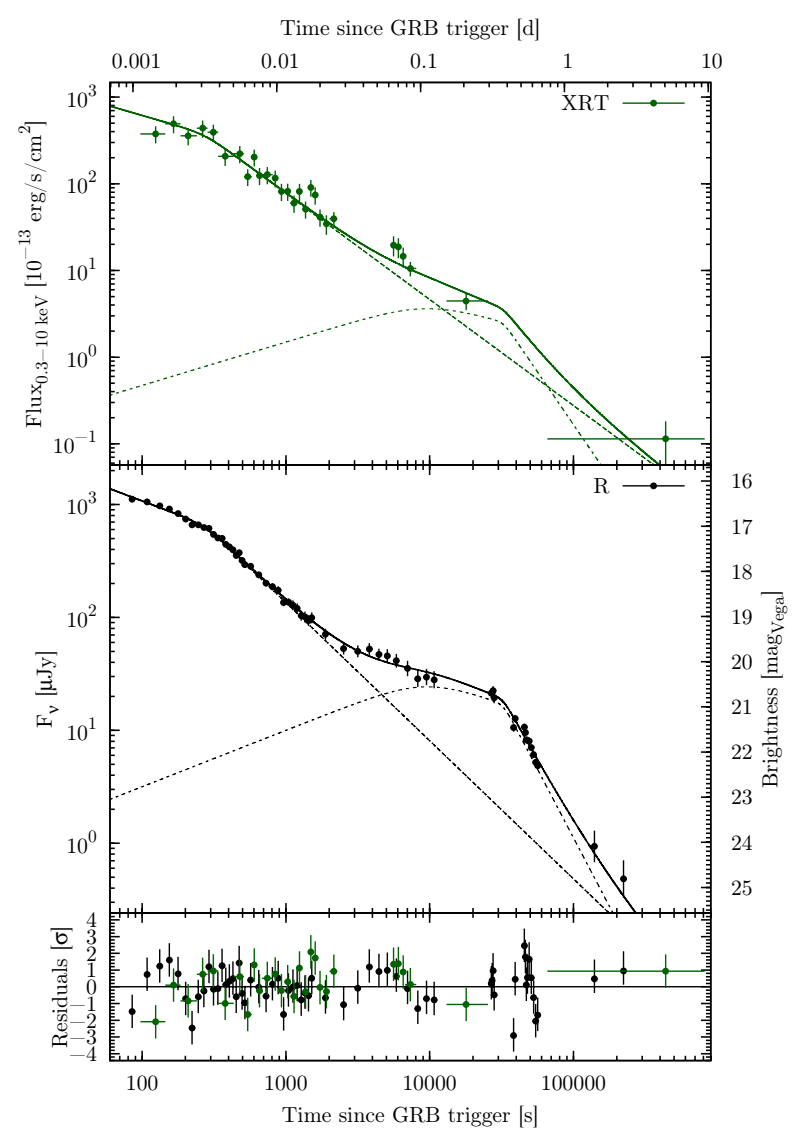

Fig. 4. Best fit of the optical afterglow using a two-component jet model. Top: X-ray light curve (0.3 to $10 \mathrm{keV}$; Evans et al. 2010). Bottom: combined $R_{\mathrm{c}}$-band data set (the same as in Fig. 3 ).

0.2 days the $\mathrm{X}$-ray data do not constrain the corresponding fit very much.

The flat decay of the light curve after its first break at $t=t_{\mathrm{b} 1}$ as well as the closure relations (Zhang \& Mészáros 2004) show that this first break cannot be the jet break of the narrow-jet component. A plausible explanation of this part of the light curve could the cessation of an energy injection episode (cf. Zhang et al. 2006; see also Xin et al. 2010). However, of primary interest here is only the late break of the optical light curve at $t=t_{\mathrm{b} 3}$. Regardless of the most suitable model, a single-jet model (Fig. 3) or a two-component jet model (Fig. 4), the deduced break time as well as the post-break decay slope do not change.

\subsection{The closure relations and the jet parameter}

The Swift/XRT data (Evans et al. 2010) show that for $t>10^{4} \mathrm{~s}$ the spectral slope in the X-ray band is constant with $\beta_{\mathrm{X}}=$ $0.91 \pm 0.30$. Combining this with the measured spectral slope in the optical/NIR bands (Sect. 3.1), and applying the closure relations, it follows that for $t>0.4$ days a jet model is in reasonable agreement with the observations (Table A.4). Thereby, a jet model with sideways expansion is preferred.

Following Sari et al. (1999), the jet half-opening angle $\Theta_{\text {jet }}^{\mathrm{ISM}}$ for an ISM environment is $\Theta_{\text {jet }}^{\mathrm{ISM}}=\frac{1}{6}\left(\frac{t_{\mathrm{b}}}{1+z}\right)^{3 / 8}\left(\frac{n_{0} \eta_{\gamma}}{\kappa E_{52}}\right)^{1 / 8}$, where, $E_{52}$ is the isotropic equivalent energy of the prompt emission in units of $10^{52} \mathrm{erg}, n_{0}$ is the density of the ambient medium in $\mathrm{cm}^{-3}, \eta_{\gamma}$ is the efficiency of the shock in converting the energy of the ejecta into gamma radiation, and $t_{\mathrm{b}}$ is the break time in days. The parameter $\kappa$ is 1.0 for a single-jet scenario, while within the context of the two-component jet model the isotropic equivalent energy of the wide jet is around $10 \%$ of 
the corresponding number for the narrow-jet component (Peng et al. 2005), i.e. it is $\kappa=0.1$. Using $n_{0}=10 \mathrm{~cm}^{-3}$ (Xin et al. 2010) and $\eta_{\gamma}=0.2$, for the observed break time at $t_{\mathrm{b}}=$ 0.4 days with $E_{52}=0.42_{-0.04}^{+0.59}$ (Levesque et al. 2010), it follows that $\Theta_{\text {jet }}^{\mathrm{w}}=(6.5 \pm 0.4)$ degrees and $E_{\gamma}^{\mathrm{corr}, \mathrm{w}}\left[1\right.$ to $\left.10^{4} \mathrm{keV}\right]=$ $(4.2 \pm 1.4) \times 10^{48} \mathrm{erg}$. Within the framework of the single-jet scenario, the break time is basically the same (Fig. 3), $\kappa=1$, and it follows that $\Theta_{\text {jet }}=(4.8 \pm 0.3)$ degrees, as well as $E_{\gamma}^{\text {corr }}=(2.3 \pm 0.8) \times 10^{49} \mathrm{erg}$.

Before the suspected jet break at 0.4 days, the observed light curve decay between about 0.1 and 0.4 days is quite shallow $\left(\alpha_{4} ;\right.$ Fig. 4$)$, while the closure relations in this case, for a model with isotropic expansion, predict a steeper decay $(\alpha>1)$. We caution, however, that the lack of data in this evolutionary phase makes it impossible to draw definite conclusions here.

\subsection{The afterglow compared to other short bursts}

Some authors have proposed (e.g., Zhang et al. 2009) a phenomenological classification based on the link with the progenitor, defining type II in the case of a collapsar event and type I in the case of merging compact objects, independent of the actual duration of the GRBs.

Using the methods detailed in Kann et al. (2006), we created a composite light curve of the afterglow of GRB 090426 and compared it to the afterglow samples of Kann et al. (2010, type II GRBs), and Kann et al. (2011, type I GRBs). Observationally, the afterglow is seen to lie in the faint end of the distribution of type II GRB afterglows (Fig. A.1), especially in cases of late steep decay, and there are several type I GRB afterglows that are brighter (e.g., GRB 050724, Berger et al. 2005; GRB 051221A, Soderberg et al. 2006; GRB 060614, Della Valle et al. 2006; Fynbo et al. 2006; Gal-Yam et al. 2006; Mangano et al. 2007) at this time. However, GRB 090426 lies at a much higher redshift $(z=2.6)$ than the aforementioned GRBs $(z=0.1-0.5)$. In the $z=1$ system (Fig. A.2), the afterglow of GRB 090426 is clearly seen as an average afterglow relative to the type II GRB afterglow sample. At $t=1$ day (in the $z=1$ frame), it would have had an absolute brightness of $M_{B}=-22.02 \pm 0.35$, which is just one magnitude fainter $(2 \sigma)$ than the mean of the afterglow comparison sample based on Swift detections (Kann et al. 2010). On the other hand, the afterglow of GRB 090426 is at all times more luminous than any afterglow of the type I sample except for the controversial case of GRB 060121 (Kann et al. 2011). There is a strong indication that, in spite of its very short duration, GRB 090426 is a type II GRB, in accordance with other studies (Zhang et al. 2009; Levesque et al. 2010; Xin et al. 2010).

\section{Summary and conclusions}

We have presented TLS/GROND data of the optical/NIR afterglow of GRB 090426, which show that the afterglow features a second break that was missed in all previously published data sets. On the basis of its achromaticity in the optical/NIR bands and in agreement with the closure relations, we have argued that the late light curve break at 0.4 days is a jet break. Its calculated half-opening angle agrees well with the distribution of halfopening angles found for long bursts. In addition, the observed luminosity of the afterglow also suggests that GRB 090426 was related to a collapsar event. The interesting question then is whether the short duration of GRB 090426 in its host frame at
$z=2.609\left(T_{90}=0.33 \mathrm{~s}\right)$ can be explained within the framework of the collapsar model and how this compares to other long bursts of similar short duration in their host frame (Greiner 2011). More observational data of other short burst afterglows are needed, not only to derive more reliable statistics but also to understand wether this short burst is an exception rather than the rule.

In a recent paper, Thöne et al. (2011) find further arguments that GRB 090426 was due to a collapsar event.

Acknowledgements. A.N.G. acknowledges useful discussions with Francisco Molleda Sanchez (Madrid, Spain) and Manuel Segura Morales (La Laguna, Spain). A.N.G., D.A.K., A. Rossi, \& S.K. acknowledges support by grant DFG K1 766/16-1. A.N.G., A. Rossi \& A.U. are grateful for travel funding support through MPE. A. Rossi acknowledges support from the BLANCEFLOR Boncompagni-Ludovisi, née Bildt foundation, T.K. by the DFG cluster of excellence 'Origin and Structure of the Universe', F.O. funding of his Ph.D. through the DAAD, M.N. support by DFG grant SA 2001/2-1 and P.S. by DFG grant SA 2001/1-1. Part of the funding for GROND (both hardware and personnel) was generously granted from the Leibniz-Prize to G. Hasinger (DFG grant HA 1850/28-1). This work made use of data supplied by the UK Swift science data center at the University of Leicester. We thank the referee for very helpful remarks.

\section{References}

Antonelli, L. A., D’Avanzo, P., Perna, R., et al. 2009, A\&A, 507, L45 Berger, E. 2010, ApJ, 722, 1946

Berger, E., Kulkarni, S. R., Pooley, G., et al. 2003, Nature, 426, 154 Berger, E., Price, P. A., Cenko, S. B., et al. 2005, Nature, 438, 988 Beuermann, K., Hessman, F. V., Reinsch, K., et al. 1999, A\&A, 352, L26 Burrows, D. N., Grupe, D., Capalbi, M., et al. 2006, ApJ, 653, 468 Della Valle, M., Chincarini, G., Panagia, N., et al. 2006, Nature, 444, 1050 Evans, P. A., Willingale, R., Osborne, J. P., et al. 2010, A\&A, 519, A102 Filgas, R., Krühler, T., Greiner, J., et al. 2011, A\&A, 526, A113 Fong, W., Berger, E., \& Fox, D. B. 2010, ApJ, 708, 9

Frail, D. A., Kulkarni, S. R., Sari, R., et al. 2001, ApJ, 562, L55 Fynbo, J. P. U., Watson, D., Thöne, C. C., et al. 2006, Nature, 444, 1047 Gal-Yam, A., Fox, D. B., Price, P. A., et al. 2006, Nature, 444, 1053 Gehrels, N., Chincarini, G., Giommi, P., et al. 2004, ApJ, 611, 1005 Gehrels, N., Ramirez-Ruiz, E., \& Fox, D. B. 2009, ARA\&A, 47, 567 Greiner, J. 2011, in press [arXiv: 1102 . 0472]

Greiner, J., Bornemann, W., Clemens, C., et al. 2008, PASP, 120, 405 Greiner, J., Krühler, T., Klose, S., et al. 2011, A\&A, 526, A30 Grupe, D., Burrows, D. N., Patel, S. K., et al. 2006, ApJ, 653, 462 Kann, D. A., Klose, S., \& Zeh, A. 2006, ApJ, 641, 993 Kann, D. A., Klose, S., Zhang, B., et al. 2011, ApJ, 734, 96 Kann, D. A., Klose, S., Zhang, B., et al. 2010, ApJ, 720, 1513 Krühler, T., Küpcü Yoldaş, A., Greiner, J., et al. 2008, ApJ, 685, 376 Levesque, E. M., Bloom, J. S., Butler, N. R., et al. 2010, MNRAS, 401, 963 Mangano, V., Holland, S. T., Malesani, D., et al. 2007, A\&A, 470, 105 McBreen, S., Krühler, T., Rau, A., et al. 2010, A\&A, 516, A71 Nakar, E. 2007, Phys. Rep., 442, 166

Nysewander, M., Fruchter, A. S., \& Pe'er, A. 2009, ApJ, 701, 824 Olivares, F., Küpcü Yoldaş, A., Greiner, J., \& Yoldaş, A. 2009, GCN, 9268 Panaitescu, A., \& Kumar, P. 2000, ApJ, 543, 66

Peng, F., Königl, A., \& Granot, J. 2005, ApJ, 626, 966 Racusin, J. L., Karpov, S. V., Sokolowski, M., et al. 2008, Nature, 455, 183 Racusin, J. L., Liang, E. W., Burrows, D. N., et al. 2009, ApJ, 698, 43 Sari, R., Piran, T., \& Halpern, J. P. 1999, ApJ, 519, L17 Schlegel, D. J., Finkbeiner, D. P., \& Davis, M. 1998, ApJ, 500, 525 Soderberg, A. M., Berger, E., Kasliwal, M., et al. 2006, ApJ, 650, 261 Thöne, C. C., Campana, S., Lazzati, D., et al. 2011, MNRAS, 414, 479 Tody, D. 1993, ASP Conf. Ser., 52, 173

Woosley, S. E., \& Bloom, J. S. 2006, ARA\&A, 44, 507 Xin, L., Liang, E., Wei, J., et al. 2010, MNRAS, 1404 Yoldaş, A. K., Krühler, T., Greiner, J., et al. 2008, AIP Conf. Ser., 1000, 227 Zeh, A., Klose, S., \& Kann, D. A. 2006, ApJ, 637, 889 Zhang, B., \& Mészáros, P. 2004, Int. J. Mod. Phys. A, 19, 2385

Zhang, B., Fan, Y. Z., Dyks, J., et al. 2006, ApJ, 642, 354 Zhang, B., Zhang, B., Virgili, F. J., et al. 2009, ApJ, 703, 1696 


\section{Appendix A: Observational data and afterglow luminosity}

Table A.1. $\log$ of the GROND observations (in case of the first epoch data these are OBs 1 to 10), with the magnitudes given in the AB system (not corrected for Galactic extinction).

\begin{tabular}{llllllll}
\hline \hline Time $(\mathrm{s})$ & $g^{\prime}$ & $r^{\prime}$ & $i^{\prime}$ & $z^{\prime}$ & $J$ & $H$ & $K$ \\
\hline 44729 & $21.90(05)$ & $21.50(08)$ & $21.46(08)$ & $21.30(08)$ & $>21.2$ & $>20.6$ & $>19.9$ \\
45506 & $21.98(06)$ & $21.57(06)$ & $21.59(08)$ & $21.50(21)$ & $>20.9$ & $>20.2$ & $>19.8$ \\
46268 & $22.03(04)$ & $21.67(05)$ & $21.59(09)$ & $21.41(11)$ & $>21.6$ & $>20.8$ & $>19.8$ \\
47037 & $22.00(05)$ & $21.82(05)$ & $21.55(08)$ & $21.47(14)$ & $>21.6$ & $>21.0$ & $>19.9$ \\
47812 & $22.08(05)$ & $21.83(04)$ & $21.64(08)$ & $21.29(15)$ & $>21.7$ & $>21.1$ & $>20.2$ \\
49112 & $22.17(03)$ & $21.84(03)$ & $21.67(05)$ & $21.50(07)$ & $>22.1$ & $>21.4$ & $>20.4$ \\
50930 & $22.28(03)$ & $21.96(03)$ & $21.79(06)$ & $21.52(07)$ & $>22.1$ & $>21.3$ & $>20.4$ \\
52756 & $22.34(03)$ & $22.10(04)$ & $21.87(05)$ & $21.71(09)$ & $>22.2$ & $>21.5$ & $>20.5$ \\
54571 & $22.44(04)$ & $22.20(03)$ & $21.96(07)$ & $21.86(05)$ & $>22.0$ & $>21.2$ & $>20.5$ \\
56374 & $22.58(04)$ & $22.29(04)$ & $22.07(06)$ & $21.93(11)$ & $>22.2$ & $>21.6$ & $>20.4$ \\
\hline 139787 & $24.03(10)$ & $23.80(11)$ & $23.63(17)$ & $23.38(18)$ & $>22.7$ & $>21.9$ & $>21.1$ \\
222822 & $24.23(11)$ & $24.24(12)$ & $23.84(15)$ & $>24.1$ & $>22.7$ & $>21.8$ & $>21.3$ \\
\hline
\end{tabular}

Notes. These results supercede the data given in Olivares et al. (2009).

Table A.2. Log of the GROND observations for the combined OBs 1 to 5 and OBs 6 to 10, with the magnitudes given in the AB system.

\begin{tabular}{llllllll}
\hline \hline Time $(\mathrm{s})$ & $g^{\prime}$ & $r^{\prime}$ & $i^{\prime}$ & $z^{\prime}$ & $J$ & $H$ & $K$ \\
\hline 46268 & $22.05(03)$ & $21.74(03)$ & $21.65(05)$ & $21.44(07)$ & $20.96(02)$ & $20.92(20)$ & $>20.1$ \\
52723 & $22.28(02)$ & $21.99(02)$ & $21.77(03)$ & $21.62(04)$ & $21.39(09)$ & $21.23(12)$ & $>20.6$ \\
\hline
\end{tabular}

Notes. Data are not corrected for Galactic extinction.

Table A.3. Log of the TLS observations, given in the Vega system.

\begin{tabular}{lll}
\hline \hline Time(s) & $R_{\mathrm{c}}$ & $I_{\mathrm{c}}$ \\
\hline 26868 & $20.90(13)$ & - \\
27146 & $20.91(14)$ & - \\
27607 & $21.05(19)$ & - \\
28063 & $20.97(14)$ & - \\
26263 & - & $20.48(18)$ \\
\hline
\end{tabular}

Notes. Data are not corrected for Galactic extinction.

Table A.4. Predicted temporal decay slopes $\alpha$ for $t>0.4$ days for various afterglow scenarios based on the measured spectral slopes $\beta_{\mathrm{opt}}=0.76 \pm$ 0.14 and $\beta_{\mathrm{X}}=0.91 \pm 0.30$.

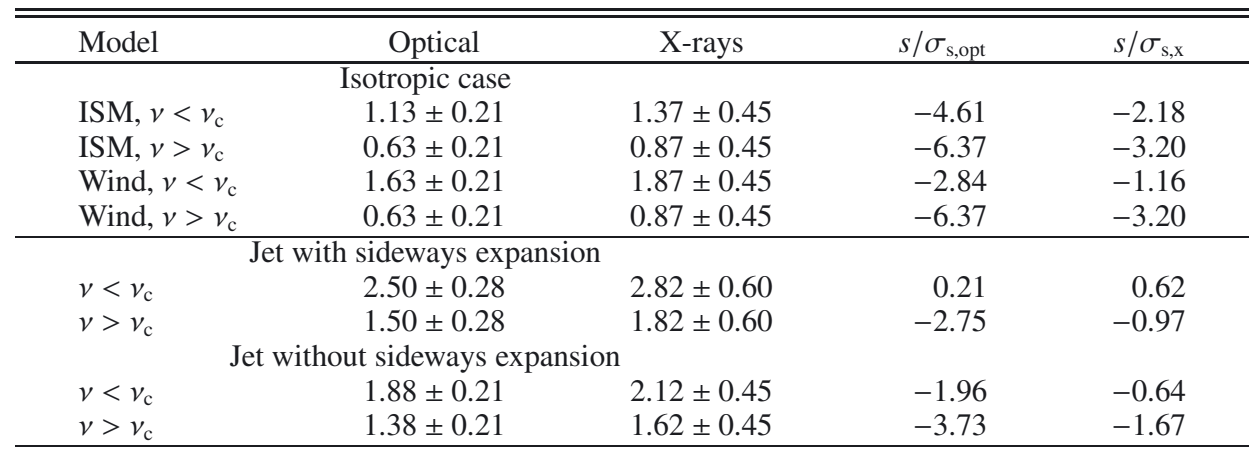

Notes. Columns 4 and 5 give the difference between the predicted and the observed ( $\alpha=2.43 \pm 0.19$; the parameter $\alpha_{5}$ in Sect. 4.1) temporal decay slope, normalized to the square root of the sum of their quadratic errors, with $s=\left(\alpha_{\text {predicted }}-\alpha_{\text {observed }}\right), \sigma_{s}^{2}=\sigma_{\text {predicted }}^{2}+\sigma_{\text {observed }}^{2}$. 


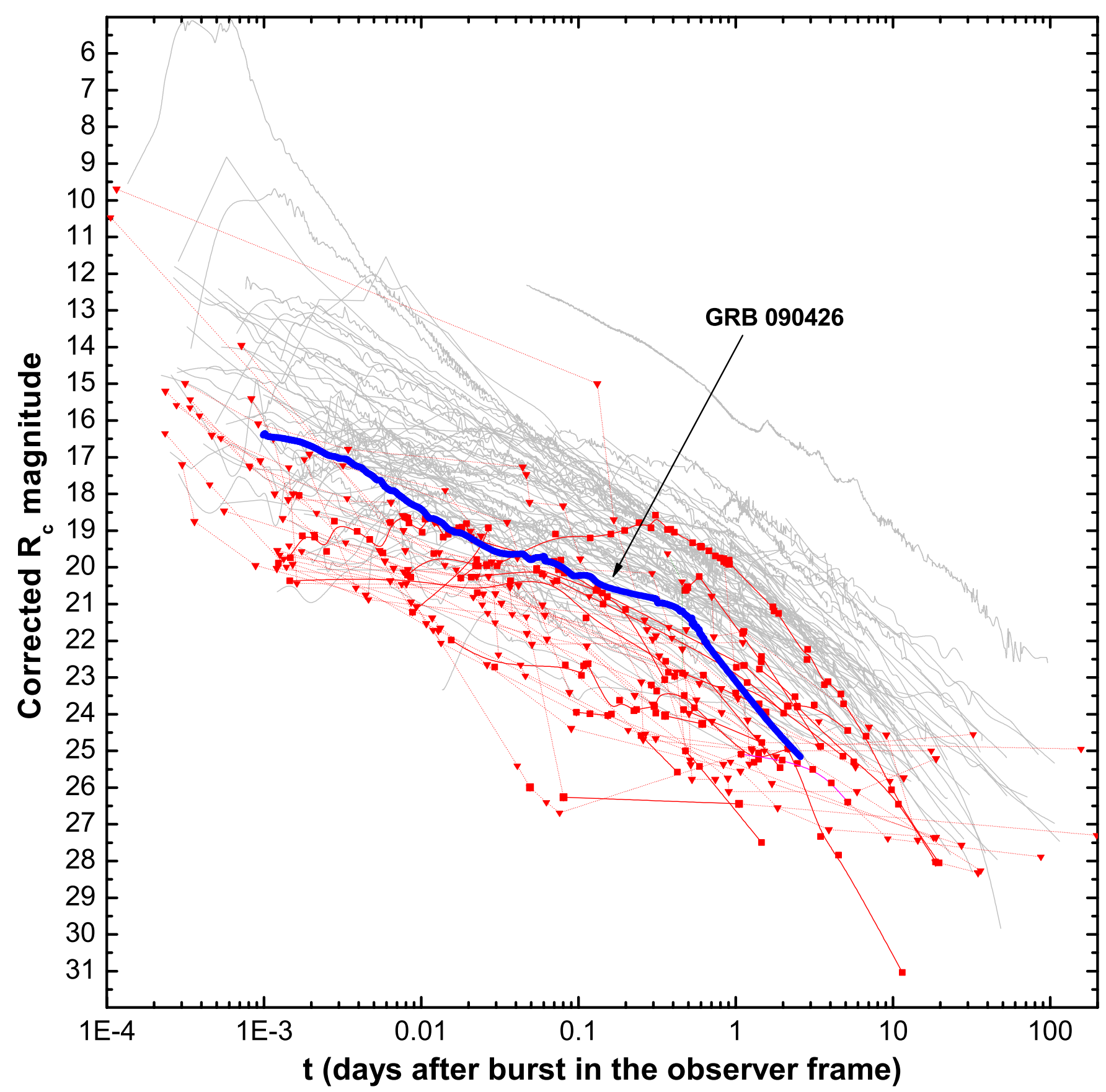

Fig. A.1. The afterglow of GRB 090426 (thick blue line) in comparison with the afterglows of type II (thing gray lines) and type I (red symbols and lines; squares connected by splines are detections, downward triangles connected by thin dashed lines are upper limits) GRBs from the sample of Kann et al. $(2011,2010)$. These afterglows have been corrected for Galactic extinction, and the host galaxy contribution has been subtracted where possible (also in the case of GRB 090426). The afterglow of GRB 090426 is seen to be among the faint type II GRB afterglows, but it is brighter than most type I GRB afterglows or limits thereon. 


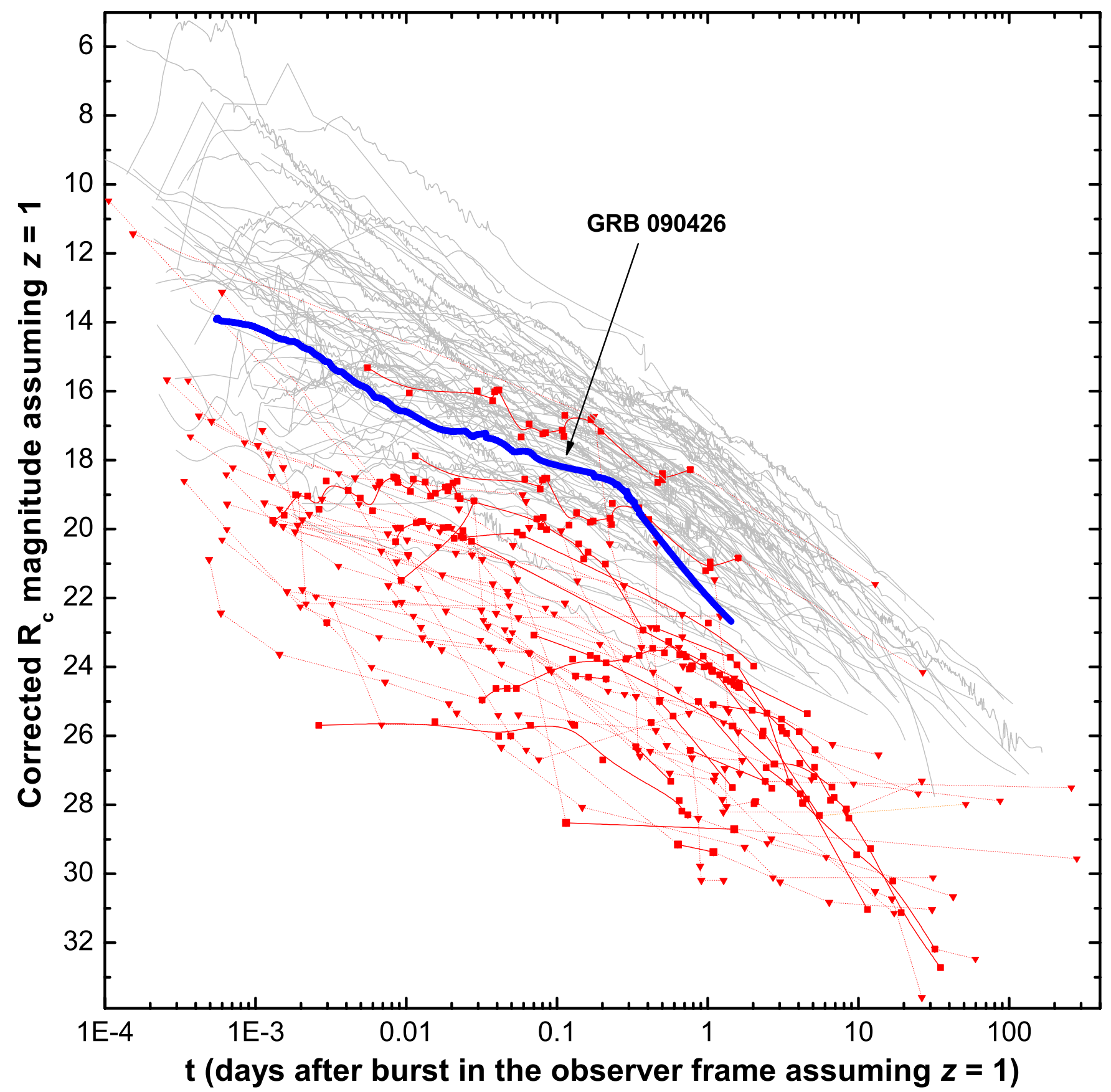

Fig. A.2. The afterglow of GRB 090426 after it has been shifted to the $z=1$ system, again in comparison with the samples (shifted using the same method) of Kann et al. (2011, 2010). The labelling is identical to that in Fig. A.1. It can now clearly be seen that the afterglow of GRB 090426 is readily comparable to the afterglow of type II GRBs (collapsar events), while it is much brighter than any type I GRB afterglow (merging compact objects), with the exception of GRB 060121, which is a controversial case. 
A\&A 531, L6 (2011)

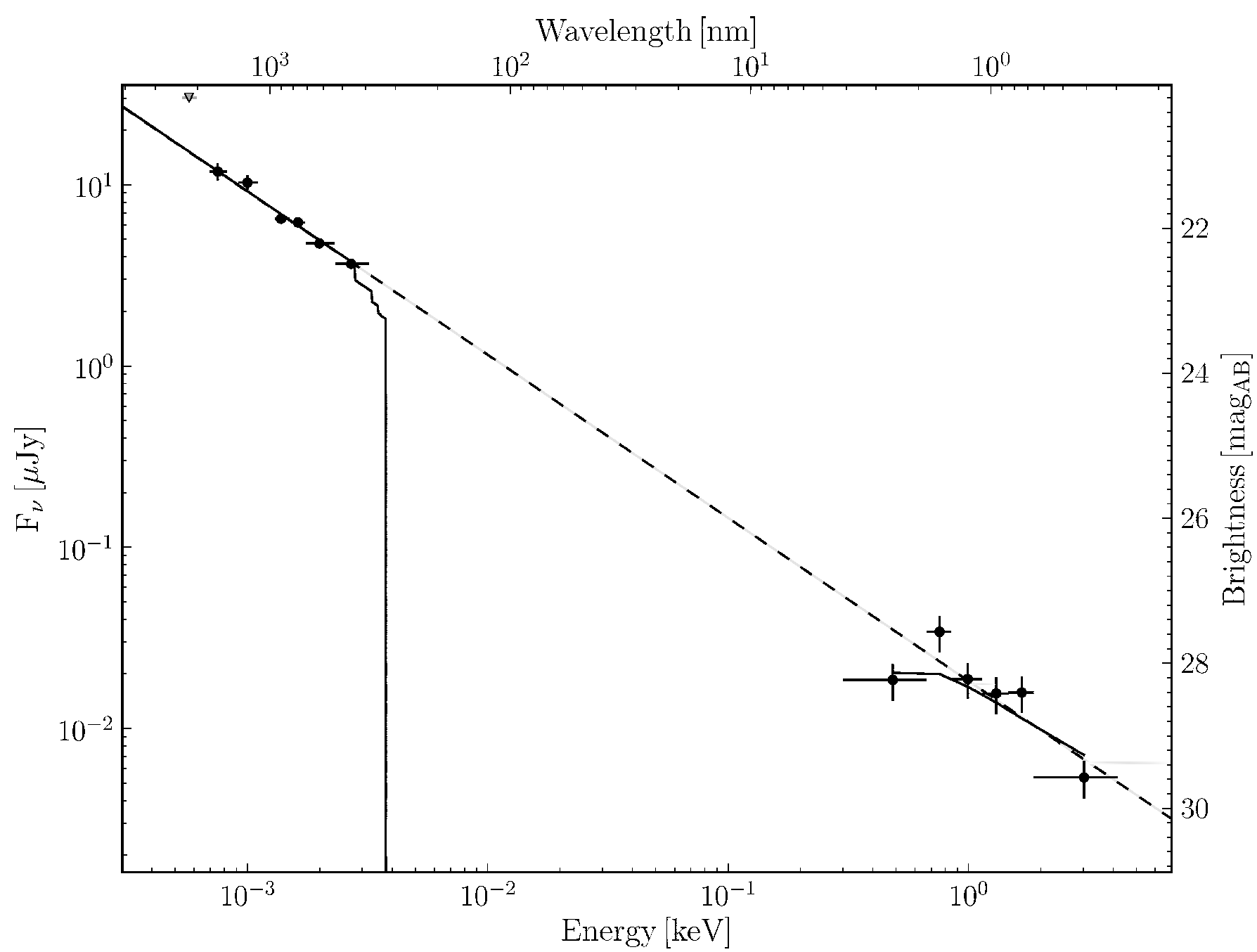

Fig. A.3. X-ray to optical spectral energy distribution of the afterglow of GRB 090426 at $t=8 \mathrm{ks}$ after the burst, calculated based on the fitted light curve (Fig. 4). The fit uses $N_{\mathrm{H}}^{\text {Gal }}=0.015 \times 10^{22} \mathrm{~cm}^{-2}$ and corresponds to a negligible host extinction, a gas column density of $N_{\mathrm{H}}^{\text {host }}=0.46_{-0.46}^{0.77} \times$ $10^{22} \mathrm{~cm}^{-2}$, and a spectral slope of $\beta_{\mathrm{OX}}=0.90 \pm 0.03\left(\chi^{2}=10.85\right.$ with 7 d.o.f., $\left.\chi_{v}=1.55\right)$. 